\title{
Les récits abrahamiques dans les traditions judaïque et islamique
}

\section{Zakaria Rhani}

\section{(2) OpenEdition}

1 Journals

Édition électronique

URL : http://journals.openedition.org/assr/13833

DOI : $10.4000 /$ assr. 13833

ISSN : $1777-5825$

Éditeur

Éditions de l'EHESS

\section{Édition imprimée}

Date de publication : 1 juin 2008

ISBN : 978-2-7132-2190-3

ISSN : 0335-5985

\section{Référence électronique}

Zakaria Rhani, «Les récits abrahamiques dans les traditions judaïque et islamique », Archives de sciences sociales des religions [En ligne], 142 I avril-juin 2008, mis en ligne le 10 décembre 2010, consulté le 10 décembre 2020. URL : http://journals.openedition.org/assr/13833 ; DOI : https:// doi.org/10.4000/assr.13833 


\section{Zakaria Rhani}

\section{Les récits abrahamiques dans les traditions judaïque et islamique}

\section{Introduction}

Ce texte tente de revoir, à la lumière de l'analyse structurale des mythes, l'histoire du prophète Abraham et de ses deux fils Ismaël et Isaac et d'essayer de suivre les transformations que cette histoire a subies en passant de la tradition juive à la tradition arabe. Je tenterai, entre autres, de démontrer comment l'ensemble des relations qui composent le récit du Patriarche, en passant d'une version à l'autre, suit une évolution symétrique mais opposée.

Le mythe, comme le souligne Jack Goody (1979: 68), est avant tout un fait social, un énoncé culturel, la clé d'un code, une fenêtre ouverte sur la structure. Deux propriétés fondamentales caractérisent la structure d'un mythe: d'une part, elle ne constitue pas le véritable sens du mythe, mais elle est ce qui le rend justement signifiant ; d'autre part, cette structure est double, à la fois historique et anhistorique (Pouillon, 1993 : 48-50). Chaque mythe est formé d'unités constitutives, qu'on nomme mythèmes, dont chacune a la nature d'une relation. Et pour être véritables, ces relations ne doivent pas être isolées, mais des ensembles de relations, et c'est seulement sous forme de combinaisons de tels ensembles que les unités constitutives acquièrent une fonction signifiante (Lévi-Strauss, 1973 : 242). L'analyse structurale consiste principalement à reconnaître et à isoler les mythèmes qui forment le mythe dans sa totalité tout en considérant l'ensemble des variantes qui le composent. En appliquant cette méthode d'analyse structurale, on parvient à ordonner toutes les variantes d'un mythe donné en une série dont les variantes placées aux deux extrémités offrent une structure symétrique mais inversée (ibid. : 257).

Le modèle anthropologique pour expliquer un mythe n'est pas donné dans le récit comme tel, au niveau conscient ; le modèle qui en définit la structure est crypté. Aussi pour décrypter un mythe faut-il lui faire subir des transformations qui laissent apparaître les oppositions fondamentales; celles-ci révéleront les dimensions cachées où s'articulent de nouvelles oppositions sur des axes sousjacents.

ARCHIVES DE SCIENCES SOCIALES DES RELIGIONS 142 (avril-juin 2008), pp. 27-46 


\section{8 - ARCHIVES DE SCIENCES SOCIALES DES RELIGIONS}

C'est bien dans la formule canonique posée par Lévi-Strauss que la structure $\mathrm{du}$ mythe prend forme; cette équation démontre l'existence d'une relation d'équivalence entre deux situations en opposition. Deux conditions sont nécessaires pour qu'une telle structure soit possible: il faut, d'une part, qu'un des termes soit remplacé par son contraire et, d'autre part, qu'une inversion corrélative se produise entre la valeur de fonction et la valeur de terme de deux éléments (ibid. : 262). Plus généralement, il s'agit d'appréhender le mythe comme un système de différence cohérent et intègre ; à ce sujet, Lévi-Strauss définit un système de différence comme étant celui qui ne conduit ni à leur simple juxtaposition, ni à leur effacement artificiel.

Une application fascinante de l'analyse structurale est donnée par un texte de Lévi-Strauss intitulé Structuralisme et écologie ${ }^{1}$ où il compare deux versions qui paraissent différentes d'un mythe indien chez les Bella Bella et leurs voisins les Chilcotins. Dans son analyse des deux variantes du mythe, l'auteur élabore une règle essentielle de l'analyse structurale : chaque fois que dans une version apparaît un détail qui semble détonner par rapport aux autres, il est vraisemblable que la version déviante s'efforce de dire l'opposé d'une version normale existant par ailleurs et généralement pas très loin de l'autre. Ainsi les deux versions seraient-elles placées dans une construction symétrique où les symboles sont généralement inversés. En fin d'analyse, Lévi-Strauss note qu'il existe des ensembles de règles, compliquées mais cohérentes, permettant de transformer un mythe en un autre. Ces règles se révèlent comme les manifestations visibles de lois qui structurent l'esprit de personnes d'un groupe entendant des voisins raconter un de leurs mythes. "Ils l'emprunteront peut-être, mais non sans le déformer par l'effet d'opérations mentales dont ils ne sont pas les maîtres. Ils adopteront le mythe pour ne pas se sentir en état d'infériorité, tout en le remaniant consciemment ou inconsciemment jusqu'à ce qu'il devienne le leur » (1983 : 151).

Il est important pour Lévi-Strauss de savoir quelle version particulière d'un mythe il va choisir comme référence pour amorcer l'analyse. Pour notre étude, quoique les deux versions étudiées - juive et musulmane - se situent dans la diachronie, il n'est pas moins difficile de décider par laquelle nous allons commencer, puisque même si la version biblique est antérieure dans le temps, la tradition postbiblique pourrait subir des transformations surtout lors des premiers contacts avec les musulmans. Dans le présent essai, je commencerai par présenter tout d'abord la version judéo-chrétienne, non parce qu'elle constitue la version normale, la tradition arabo-islamique constituant la version déviante, mais simplement pour des raisons méthodologiques et pour respecter une certaine chronologie nécessaire à la clarté de l'exposé.

1. Ce texte fut prononcé lors d'une conférence à Barnard College aux États-Unis en 1972 (Lévi-Strauss, 1983 ; Clément, 2002). 
Dans la présente étude, je ne prendrai en compte de l'histoire du Patriarche et de sa famille que des composantes nécessaires à mon analyse.

\section{La version biblique du récit abrahamique}

La lecture de Genèse 16,2 nous renseigne sur la vie du Patriarche Abraham. Sara sa femme, ne pouvant lui donner d'enfants, lui demande d'aller vers sa servante Hagar: «Et Saraï dit à Abram : voici, l'Éternel m'a rendue stérile ; viens, je te prie, vers ma servante; peut-être aurai-je par elle des enfants. Abram écouta la voix de Saraï ». Abraham eut un garçon d'Hagar qu'il nomma Ismaël. Treize ans plus tard, le Patriarche reçut l'annonce et la grâce divine d'une nouvelle naissance; mais cette fois de sa première épouse Sara. Cette annonce se manifesta par le retour du sang menstruel de celle-ci :

«L'Éternel se souvient de ce qu'il a dit à Sara, et l'Éternel accomplit pour Sara ce qu'il avait promis. Sara devint enceinte, et elle enfanta un fils à Abraham dans sa vieillesse [...]. Abraham donna le nom d'Isaac au fils que Sara lui avait enfanté. "(Genèse 21,1-3). C'est la naissance miraculeuse d'Isaac, l'enfant de la promesse, avec qui Dieu, écartant Ismaël, va faire alliance. Le signe de cette alliance est donné, non seulement par la naissance extraordinaire, mais aussi, et surtout, par le sacrifice de son fils unique, Isaac, que Dieu demande à Abraham. Le sacrifié est sauvé en dernier lieu en le remplaçant par un bélier céleste.

Après la naissance d'Isaac, l'attitude de Sara à l'égard d'Hagar et de son fils Ismaël change. "L'enfant [Isaac] grandit, et fut sevré ; et Abraham fit un grand festin le jour où Isaac fut sevré. Sara vit rire le fils qu'Agar, l'Égyptienne, avait enfanté à Abraham. Et elle dit à Abraham : Chasse cette servante et son fils, car le fils de cette servante n'héritera pas avec mon fils, avec Isaac " (Genèse 21,9-10). Face à cette demande pressante de Sara, Abraham hésite, mais finit par se résigner. Il renvoie Hagar et son fils vers le désert.

\section{La version arabo-islamique}

L'analyse du récit arabo-islamique d'Abraham ${ }^{2}$ se base surtout sur la tradition post-coranique. Car, à l'inverse de la Bible, le Coran ne détaille pas la vie du Patriarche et de sa famille.

Le Coran fait une place importante à Abraham, le père des croyants et l'ami (khalîl) d'Allah. Il est l'un des quatre grands messagers, avec Moïse, Jésus et Mohammed. Il est d'ailleurs, biologiquement parlant, le père de ces trois derniers prophètes, d'où son importance cruciale dans l'histoire de la révélation. Ce sont

2. Les équivalents arabes des noms bibliques Abraham, Sara, Hagar, Isaac et Ismaël sont respectivement Ibrâhîm (ou Ibrahâm), Sâra, Hâjar, Ishâq et Ismâ'îl. Vu leur grande similarité et pour la simplicité de l'exposé, j'adopterai les noms bibliques pour les deux versions. 
en particulier les versets révélés dans la seconde période de la vie du prophète, après son émigration de la Mecque à Médine, qui évoquent son rôle dans la tradition prophétique et la fondation de la croyance en un Dieu unique. Ces versets médinois parlent également de son fils Ismaël qui restaura à ses côtés le temple de la Kaaba.

Pour la séquence des événements caractérisant généralement la vie du Patriarche et de sa famille, je m'appuierai sur le récit qu'en donne l'historiographe arabe du IX ${ }^{\mathrm{e}}$ siècle, Mohammed Ibn Jarir al-Tabari.

«Or Abraham acquit de grandes richesses et il désira avoir de Sara un enfant : mais il n'en eut aucun. Sara dit alors à Abraham : Tu n'auras point d'enfants de moi : si tu veux, je te donnerai Agar, peut-être auras-tu d'elle un enfant. Abraham répondit : j'y consens. (...) Lorsque Agar accoucha d'Ismaël, Abraham fut rempli de joie ; mais Sara éprouva de la colère et une violente jalousie. N'étant plus maîtresse d'elle-même, elle eut des querelles et des disputes avec Abraham, et elle lui dit des injures (...) Les choses allèrent à un tel point que le cœur d'Abraham poussa des gémissements vers Dieu et se plaignit de Sara (...) Abraham resta d'abord sans savoir que faire, ni quel moyen employer dans cette conjecture. Il se leva ensuite, emmena Ismaël et Agar, prit un peu de nourriture et de boisson et se dirigea vers le désert (...) Lorsqu'il eut fait un peu de chemin, Gabriel se présenta devant lui en disant : Où vas-tu et où conduis-tu cet enfant ? Abraham répondit (...) : je les éloigne de la main de Sara. Gabriel dit à Abraham : Conduis-les dans l'enceinte consacrée à Dieu, dans l'endroit où était la maison visitée " $2003: 80-81)$.

Mais arrivé dans l'endroit indiqué, l'emplacement du sanctuaire de la Kaaba, Abraham réalisa qu'il était complètement désert. Après quelques hésitations, il finit par laisser Hagar et Ismaël dans ce lieu inhospitalier avec une outre d'eau et quelques provisions. L'eau dont ils disposaient s'épuisa, ainsi que les provisions, Hagar se mit alors à chercher désespérément de quoi apaiser la soif de son fils en courant entre les collines de Safâ et Marwâ. Ismaël se mit à pleurer et ayant frappé du talon contre terre, une source en jaillit miraculeusement ${ }^{3}$; c'est la source de Zamzam considérée comme sacrée et utilisée par les pèlerins. La tribu de Jorhom, à la recherche de sources d'eau dans la région, finit par s'établir auprès d'Hagar et son fils. L'enfant grandit parmi eux et épousa l'une des leurs. Plus tard, sur un ordre divin, Abraham se rendit à la Mecque auprès de son fils pour bâtir le temple de la Kaaba ${ }^{4}$.

Concernant le sacrifice qu'accepte de faire Abraham de son fils, le Coran en évoque quelques épisodes, mais sans que le nom du fils promis à l'immolation

3. Selon une autre version plus ancienne rapportée par 'Abdallâh Ibn 'Abbâs, exégète renommé et cousin du prophète, c'est l'archange Gabriel qui guida Hagar vers la source d'eau (cité dans Dagorn, 1981:265).

4. Tabari $(2003: 80-81,92)$. Pour un récit plus détaillé de la geste ismaélienne voir aussi Dagorn (op. cit. : 263-284). 
LES RÉCITS ABRAHAMIQUES DANS LES TRADITIONS JUDAÏQUE ET ISLAMIQUE - 3 I

soit précisé. Le Coran rapporte que le prophète Abraham eut une vision nocturne au cours de laquelle il se vit immolant son fils : " quand ce dernier parvint à l'âge actif, il [Abraham] lui dit: "Mon enfant je me suis vu en rêve t'égorger. Examine quel parti prendre ${ }^{5}$. Le Coran présente un fils totalement soumis à la volonté de Dieu : "Père, faites ce dont vous avez reçu commandement. Vous me trouverez, si Dieu veut, patient entre tous. Ayant ainsi tous deux manifesté leur soumission, il le jeta à terre sur la tempe " ${ }^{6}$. Dieu alors, pour récompenser Abraham et son fils de leur profonde foi et de leur soumission, remplaça le fils par un bélier : " alors Nous l'appelâmes : Abraham ! Tu as avéré la vision. Ainsi les bel-agissants Nous rétribuons. Ce n'était là qu'épreuve d'élucidation. Nous le rachetâmes contre une prestigieuse victime ${ }^{7}$.

Une première comparaison entre les récits biblique et coranique permet de soulever une question cruciale pour notre analyse structurale. Pourquoi ce détail, concernant le nom de la victime, si important pour le récit biblique, n'est pas évoqué par le Coran ? Il y a lieu de croire que le nom du fils promis à l'immolation n'est pas pertinent pour le récit coranique, car le sacrifice n'a pas de conséquences électives; il n'est qu'un exemple de foi et de soumission à Dieu. Il n'existe nulle mention dans le Coran que les fils d'Abraham donneront naissance à des nations ou à des peuples différents ${ }^{8}$. Le nom du fils promis au sacrifice n'est pas spécifié dans le Coran: qu'en est-il de la tradition post-coranique ? S'agit-il d'Isaac ou d'Ismaël ?

\section{Structure et sacrifice}

La première principale transformation qui s'établira dans la tradition musulmane par rapport à la version biblique du récit abrahamique concerne le nom du fils voué au sacrifice. Il s'agit d'Isaac dans celle-ci, d'Ismaël dans celle-là. Pour bien comprendre le sens de cette transformation, il est important de suivre les différentes étapes qui se sont succédées avant que le nom d'Ismaël ne soit retenu comme étant le sacrifié.

L'ambiguité du sacrifice, que le Coran n'explicite pas, n'est pas levée non plus par le hadith. Jacques Berque, dans une note explicative du verset (37, $101)^{9}$, écrit que le héros de la scène d'oblation est Ismaël. L'auteur, pour confirmer son hypothèse, s'appuie sur un hadith où le prophète Mohammed est appelé

5. Coran 37, 102, traduction Jacques Berque.

6. Ibid.

7. Ibid., 103-107.

8. À ce sujet, Kaltner écrit : "there is no suggestion in the Qur'an of a chosen people set apart to enjoy a special relationship with Allah. Islam maintains that all people are born Muslims ("sublitters" to the divine will). It is impossible for Allah to choose some for salvation while excluding the rest » (2002: 23).

9. Avant le récit du sacrifice, il est dit : "Nous lui [Abraham] fîmes donc l'annonce d'un garçon longanime». 
par un bédouin "fils des deux égorgés ". Ce hadith laisse supposer que c'est Ismaël qui était destiné au sacrifice. Le second immolé n'est autre que le propre père de Mohammed, 'Abdallâh ${ }^{10}$. L'authenticité de ce hadith reste toutefois suspecte puisque d'autres hadiths désignent également Isaac comme la victime ${ }^{11}$. Ainsi les deux grands rapporteurs de hadiths que sont Ibn 'Abbâs et Abû Hûrayra sont invoqués indistinctement en faveur des deux fils d'Abraham.

La position des chroniqueurs et des commentateurs sur la question du sacrifice n'est pas homogène non plus, elle est très divergente. Voici le résumé du récit que donne al-Tabari de l'immolation manquée : lorsque Abraham demanda un enfant à Dieu, il fit le vœu de le lui sacrifier. Des années s'écoulèrent après la naissance d'Ismaël et d'Isaac et Abraham n'avait toujours pas tenu sa promesse. C'est alors qu'il vit Dieu en songe lui demandant d'accomplir son vœu. "Quant à la personne qui fut immolée, ajoute al-Tabari, on n'est pas d'accord sur ce point. Suivant les uns ce fut Ismaël qu'Abraham offrit en sacrifice, et suivant les autres ce fut Isaac. Or nous avons deux traditions, qui viennent à l'appui de ces deux opinions différentes" $(2003: 88)$.

Al-Tabari (2005) mentionne dix-sept sources en faveur d'Isaac et vingt et une en faveur d'Ismaël. Ibn Qutayba (1969) ${ }^{12}$, auteur et historien du IX ${ }^{\mathrm{e}}$ siècle, cite cinq listes de garants en faveur d'Isaac et deux seulement en faveur d'Ismaël. Les deux historiographes se prononcent quant à eux en faveur d'Isaac ${ }^{13}$. À l'époque de Mohammed et ses califes, particulièrement Omar Ibn al-Khattâb et 'Ali Ibn Abî Tâlib, l'opinion dominante était en faveur d'Isaac (Dagorn, 1981 ; Kaltner, 2002). C'est surtout la tradition musulmane tardive qui va dissiper irrémédiablement cette ambiguité et attribuer, sans le moindre doute, le rôle du sacrifié à Ismaël ${ }^{14}$. Les raisons de ce déplacement sont difficiles à déterminer avec précision, mais tout laisse à croire que ce rôle de "sacrifié » a un rapport étroit avec la ré-élaboration des généalogies arabes aux époques omeyyades et

10. 'Abdallâh a été aussi promis à l'immolation par son père 'Abdalmûttalib, mais il a finalement été racheté contre le sacrifice de cent camélidés présentés comme offrande aux divinités préislamiques de la Mecque.

11. Parmi ceux qui acceptent l'authenticité de cet échange entre le bédouin et le prophète, certains identifient tout de même le premier égorgé à Isaac et non à Ismaël puisque selon la tradition arabe l'oncle paternel est aussi considéré comme père. Et pour d'autres récits en faveur d'Isaac, voir (Dagorn, 1981 : 358-359).

12. Ibn Qutayba, en introduction à son chapitre sur Isaac fils d'Abraham, affirme que la majorité des exégètes musulmans soutient que c'est bien Isaac l'immolé (1969: 35).

13. Il est intéressant d'apporter ici le commentaire de l'éditeur de la dernière version arabe de l'Histoire d'al-Tabari. Iyyad al-Qayssi écrit : l'avis de l'historiographe arabe est une erreur évidente car l'immolé est Ismaël et non Isaac. Cette position est celle des fils d'Israël qui ont volontairement instauré Isaac comme sacrifié. Car ils savaient que de la descendance de celuici sortirait le prophète des derniers temps. Ils veulent ainsi que celui-ci soit des leurs. Les compagnons du prophète Mohammed, ajoute-t-il, ont repris les opinions des Israélites sans connaître leurs objectifs odieux (al-Tabari, $2005: 165$ ).

14. Pour tous les interlocuteurs arabes que j'ai interrogés sur le fils d'Abraham porté au sacrifice, le nom d'Isaac n'est même pas soupçonné. 
abbassides, deux siècles après l'époque du prophète ${ }^{15}$. C'est à cette époque aussi que l'ascendance ismaélienne des Arabes a été confirmée. Le changement dans le nom du fils sacrifié reflète probablement les tensions qui caractérisaient à cette époque les relations entre Arabes et Perses qui, eux, se réclamaient d'Isaac, comme victime (Dagorn, 1981 ; Benslama, 2002 ; Kaltner, 2002) ${ }^{16}$. Ceci pourrait, sans doute, expliquer les raisons de ce déplacement du nom de la victime promise à l'immolation d'Isaac vers Ismaël. Tout se passe comme si par ce sacrifice on sanctifiait irrévocablement la filiation ismaélienne.

Reconsidérons maintenant la version biblique du sacrifice. Dans Genèse 22,2, on lit : "Dieu dit [à Abraham] : Prends ton fils, ton unique, celui que tu aimes, Isaac ». S'il est vrai que le chapitre précédent, Genèse 21,21, relate l'installation d'Ismaël dans le désert, cette séparation géographique, comme le rappelle judicieusement Römer (1999: 162), n'indique nullement qu'il cesse d'être le fils premier-né d'Abraham. Tout se passe comme si le narrateur voulait ignorer ou oublier Ismaël. La tradition rabbinique, affirme Römer, a bien ressenti le problème de l'ordre divin dans Genèse 22 et a "inventé » le dialogue suivant : "Prends ton fils. - Lequel ? demande Abraham. J'en ai deux. - Ton unique. L'un est l'unique pour sa mère et l'autre est unique pour la sienne. - Celui que tu aimes. - J'aime celui-ci et j'aime celui-là. - Isaac ». Cette transformation, bien qu'elle ait permis au Midrash de rappeler qu'Abraham avait deux fils et non un seul, ne permet toutefois que partiellement de réhabiliter Ismaël en l'insérant dans un récit dialogique entre le Père et Dieu. Ismaël n'est inclus dans le récit de la paternité que pour être exclu de l'alliance. "Apparemment, écrit Römer, il est très difficile d'accepter cette double paternité d'Abraham telle que la Bible nous la présente. Selon l'interprétation juive et chrétienne des récits de la Genèse, c'est Isaac qui apparaît toujours comme le “vrai” fils d'Abraham » (1999: 162). Vrai, peut-être! Mais est-ce qu'il est l'unique ? Sur un ordre chronologique, Isaac ne peut en aucun cas être l'unique de son père ; tandis qu'Ismaël l'était avant la naissance de son frère. La progression du récit du sacrifice de ton "fils unique » à «Isaac », pourrait être lue comme une forme d'inversion par précision ${ }^{17}$.

Après avoir présenté ces déplacements concernant le nom du fils sacrifié, j'aimerais maintenant préciser une autre transformation qui se rapporte, cette fois-ci, au lieu du sacrifice. Dans le récit biblique, la désignation topographique du lieu du sacrifice est assez explicite: «Dieu mit Abraham à l'épreuve, et lui dit : Abraham ! Il répondit : me voici ! Dieu dit : Prends ton fils, ton unique, que

15. Au fur et à mesure que l'on s'éloigne de l'époque du Prophète et des premiers musulmans, l'usage du nom d'Ismaël, ainsi que celui de sa mère Hagar, s'amplifiera. Cette question est largement analysée par René Dagorn (1981) tout au long de son livre sur la geste d'Ismaël.

16. Voir infra.

17. Il faut préciser ici que cette inversion ne représente pas un déplacement par rapport à la tradition musulmane, mais, comme on le verra ci-dessous, par rapport à l'esprit du texte biblique lui-même. 
tu aimes, Isaac; va-t-en au pays de Morija, et là tu l'offriras en holocauste sur une montagne que je te dirai " (Genèse 22,1-2). Bien que la localisation au pays de Morija ne soit pas attestée par les anciennes versions et ne soit appuyée expressément par aucune des versions bibliques, certains textes hébreux l'identifient avec la montagne sainte où s'élève le temple de Jérusalem ${ }^{18}$.

Contrairement à la Bible, le texte coranique ne fait aucune allusion au lieu de l'immolation. Mais dans la tradition post-coranique, l'idée que le sacrifice ait eu lieu à la Mecque a été largement acceptée. Ceci est probablement dû au fait qu'encore aujourd'hui on continue à perpétuer le sacrifice du Patriarche dans le cadre du sanctuaire mecquois. De ce sacrifice et ses symboles, nous déduisons qu'il y a eu un double déplacement : une inversion des personnes et une transformation des lieux (fig. 1).

\begin{tabular}{|c|c|c|}
\hline Version & Judaïque & Arabo-islamique \\
\hline Sacrifié & Fils unique $(-) \rightarrow$ Isaac $(+)$ & NI/Isaac $(-) \rightarrow$ Ismaël $(+)$ \\
\hline Lieu du sacrifice & Morija $\rightarrow$ Jérusalem $(+)$ & NI $\rightarrow$ La Mecque $(+)$ \\
\hline
\end{tabular}

Fig. 1 : les signes $(-)$ et $(+)$ sont respectivement des valeurs négatives et positives. Le NI désigne une personne ou un lieu non identifiés et la flèche représente le sens d'une inversion.

\section{Circoncision et alliance}

L'épisode de la circoncision est relaté dans Genèse 17,9-14 : « et Dieu dit à Abraham : et toi, tu garderas mon alliance, toi et la semence après toi, en leurs générations. Que tout mâle d'entre vous soit circoncis. Et vous circoncirez la chair de votre prépuce, et ce sera signe d'alliance perpétuelle. Et le mâle qui n'aura point été circoncis en la chair de son prépuce, cette âme sera retranchée de ses peuples : il aura violé mon alliance. Puis Abraham prit Ismaël, son fils. Il circoncit la chair de leur prépuce en ce même jour comme Dieu le lui avait dit. Abraham était âgé de quatre-vingt-dix-neuf ans lorsqu'il fut circoncis en la chair de son prépuce et Ismaël, son fils, était âgé de treize ans ».

Si le Coran ne fournit aucune indication à propos de la circoncision, la tradition musulmane post-coranique en fait, quant à elle, le symbole d'une autre relation bien particulière, après celle du sacrifice, entre le père et son fils. D'ailleurs sacrifice et circoncision sont souvent mis en corrélation. Il est intéressant à cet égard de remarquer que certains exégètes musulmans situent les deux épisodes sacrificiels dans la même année, et juste un an avant la naissance d'Isaac. L'anthropologue

18. 2 Chroniques 3,1 . 
Abdelsalam pense la circoncision d'Ismaël et d'Abraham comme un acte compensatoire du sacrifice manqué du fils. "Ibrahim, écrit-il, n'ayant pas sacrifié son fils mais un mouton a, en échange, offert son propre prépuce et celui d'Ismail, en signe d'alliance avec Dieu » (1999: 365).

Cette corrélation entre les deux rituels sacrificiels est toujours opérationnelle dans le monde arabo-musulman. Au Soudan, par exemple, le jour de sa circoncision, chaque garçon a le prépuce tranché en même temps qu'est égorgé un mouton. "Ainsi, ce jour commémore le souvenir d'Ismail, et renouvelle l'épreuve du sacrifice au cours duquel on ne tranche pas la gorge mais seulement le prépuce du jeune garçon" (ibid. : 366) ${ }^{19}$.

De ce qui précède, on pourrait déduire que sacrifice et circoncision sont deux pratiques mises dans un rapport symétrique; elles symbolisent l'alliance avec Dieu par un acte sanglant. Mais elles sont aussi dans une relation d'opposition (fig. 2).

\begin{tabular}{|c|c|c|c|}
\hline & Circoncision & Sacrifice & Alliance \\
\hline Isaac & + & + & + \\
\hline Ismaël & \pm & - & - \\
\hline
\end{tabular}

Fig. 2a : selon la tradition juive, le sacrifice d'Isaac est la négation de l'alliance d'Ismaël par la circoncision. Le signe $( \pm)$ sert à rappeler la circoncision d'Ismaël à treize ans, contrairement à celle d'Isaac à l'âge de huit ans.

\begin{tabular}{|c|c|c|c|}
\hline & Circoncision & Sacrifice & Alliance \\
\hline Ismaël & + & + & + \\
\hline Isaac & + & - & - \\
\hline
\end{tabular}

Fig. $2 \mathrm{~b}$ : dans la tradition musulmane, le sacrifice d'Ismaël est la confirmation de la première alliance faite par la circoncision.

Si, selon la tradition musulmane, le sacrifice d'Ismaël peut être considéré comme une confirmation de la première alliance marquée par la circoncision, le sacrifice d'Isaac, dans la tradition juive, est en quelque sorte l'annulation de la première alliance d'Ismaël faite par la circoncision. Cette hypothèse est bien confirmée par un passage rabbinique : "Ismaël se vantant du mérite qu'il avait à endurer la circoncision déjà âgé de treize ans plutôt qu'à huit jours comme Isaac, celui-ci se déclare prêt à donner sa vie en témoignage d'obéissance à Dieu »

19. Ce rituel se pratique dans d'autres pays du monde arabe. Ainsi au Maroc, par exemple, les garçons sont généralement circoncis le septième jour après leur naissance ; à cette occasion un mouton est également égorgé. 
(de Menasce, 1951 : 100). L'alliance d'Isaac par le sacrifice est perçue, non seulement, comme plus importante que l'alliance d'Ismaël faite par la circoncision, mais elle en est l'abrogation. Dans certains cas, comme dans le Livre des Jubilés, c'est l'âge même de la circoncision d'Ismaël, à treize ans, qui est évoqué pour l'exclure de toute alliance possible ${ }^{20}$.

Mais ces interprétations exclusivistes, comme le rappelle Römer (1999), vont à l'encontre du texte de la Genèse où le personnage d'Ismaël revêt un caractère important, voire principal. Non seulement la Genèse relate l'histoire de la naissance d'Ismaël et explique le sens de son nom, mais elle l'inclut aussi dans l'alliance qu'établit Dieu avec Abraham. Le récit relate l'intervention divine aussi bien pour sauver Ismaël et sa mère d'une mort certaine que pour la promesse d'une grande descendance. Mais bien que ce soit la circoncision qui permet de définir le lien étroit qui unira Ismaël et Isaac, l'alliance divine sera accordée essentiellement à ce dernier (Genèse 17,21). "C'est presque un paradoxe, écrit Römer, dans la mesure où Ismaël participe au signe de l'alliance » $(1999: 170)^{21}$. Tout se passe comme si Ismaël, bien qu'il partage le même symbole de l'alliance qu'Isaac, n'était que l'héritier d'une promesse partielle qui ne concerne que la multiplication de sa descendance ${ }^{22}$.

Souvent, c'est aussi l'ascendance agarienne d'Ismaël qui est rappelée pour l'écarter de toute alliance. Les positions différentes d'Ismaël et d'Isaac doivent donc être interprétées non seulement par rapport à leur statut respectif mais aussi en fonction de celui de leurs mères distinctes.

\section{Épouse et servante}

Paul, dans l'Épître aux Galates 4,22-26, développe une exégèse surprenante de la différence entre les deux fils d'Abraham ainsi que de leurs mères respectives : " il est écrit qu'Abraham eut deux fils, un de la femme esclave, et un de la femme libre. Mais celui de l'esclave naquit selon la chair, et celui de la femme

20. Le livre des Jubilés, écrit Millar, "retells the story of Abraham, Sarah, Hagar and Ishmael, the Covenant and the circumcision of Isaac. It also records that Ishmael was then circumcised too [...]. But it continues by proclaiming with great emphasis that the Covenant was to be solely for those circumcised at eight days, and categorically excludes Ishmael and his descendants» (1993: 37).

21. Wyatt (1996) explique ce paradoxe par une rédaction tertiaire où le récit de la promesse faite à Ismaël aurait probablement été raccroché, après sa circoncision, à une version primitive dans laquelle il a été rejeté parce que c'est Isaac qui était l'enfant promis.

22. Même la théophanie et ses conséquences électives, Genèse 16,10 , sont parfois contestées. Selon Reis, Hagar, à l'opposé de Sara et d'Abraham, n'a pas pu reconnaître le Seigneur qui a choisi les juifs pour être son peuple. "Until this moment, ajoute-t-elle, Hagar had the opportunity to be the mother of the covenantal child; now, though her son is the seed of Abraham, mother and child will have a different future. And, as pagan, the Lord will appear to Hagar and Ishmael henceforth only as Elohim » (2000:94). 
libre naquit en vertu de la promesse. Ces faits ont une valeur allégorique; car ces femmes sont deux alliances. L'une du mont Sina, enfantant pour la servitude, c'est Agar - car Agar, c'est le mont Sina en Arabie - et elle correspond à la Jérusalem actuelle, qui est dans la servitude avec ses enfants. Mais la Jérusalem d'en haut est libre, c'est notre mère [Sara] ».

Hagar, l'esclave, est donc l'allégorie de l'alliance du désert, une alliance qui a instauré l'esclavage de la Loi, alors que Sara, la femme libre, symbolise la nouvelle alliance, libératrice et qui préfigure les chrétiens. Selon Pabst (2003), ce sont les pères de l'Église qui furent les premiers à interpréter le couple Sara/ Hagar comme une allégorie du dedans et du dehors. Si Sara symbolise le dedans positif et identificateur, Hagar, quant à elle, représente l'altérité rejetée et expulsée. "Hagar, écrit Pabst, takes over the symbolism of the negative "other", the outside which is perceived as dangerous and has to be subjected. She becomes the "allegory" of foreign territory. Sarah takes the symbolism of the positive inside which is perceived as stabilizing order » $(2003: 8)$.

Dans la tradition rabbinique, le couple duel Sara/Hagar représente aussi une allégorie du même et de l'autre. Si Sara représente Jérusalem et Israël, Hagar symbolise l'étrangeté et l'altérité dans son absolu (Pabst, 2003). Zucker (1990: 44) confirme pour sa part le rôle négatif souvent assigné à Hagar dans certaines sources juives classiques. Celle-ci, affirme-t-il, est non seulement inculpée exclusivement dans le conflit qui l'a opposée à Sara, mais elle est aussi rabaissée au statut d'idolâtre et de païenne ${ }^{23}$.

Si l'on se réfère à Genèse 16,3, on remarque que les termes "femme " et " mari », désignant Saraï et Abram, sont martelés d’une façon répétitive : "Alors Sarai, femme d'Abram, prit Agar, l'Égyptienne, sa servante, et la donna pour femme à Abram, son mari... ». Pour Reis, ce caractère répétitif du verset a pour but de désigner, haut et fort, Sara comme seule femme du Patriarche. Servante et esclave, Hagar est acculée, quant à elle, au statut de mère porteuse. "As a birthing mother, écrit Reis, Hagar will have only a generative function ; once pregnant, she will lose her copulation privileges. By sleeping with Abram until she conceives, Hagar does not attain wifely status equal to Sarai's and, as Sarai's servant, the handmaid remains under her authority after conception " $(2000: 79)^{24}$.

Aux antipodes de ces interprétations négatives, plusieurs auteurs ont récemment montré la centralité de la figure hagarienne dans la Genèse (Dozeman, 1998 ; Nikaido, 2001 ; Wénin, 2001 ; Jarrel, 2002 ; Pabst, 2003). Selon Wénin,

23. Reis, dans un article très récent, réserve à Hagar le même rôle négatif. Selon elle, la réaction cruelle de Sarah contre Hagar est légitime dans la mesure où c'est cette dernière qui, après avoir rempli son rôle de mère " porteuse ", continue à s'introduire dans la tente d'Abraham. "The Handmaid, écrit-elle, wrongly but understandably, looks to Abram for comfort and for the satisfaction of her sexual appetite $"(2000: 86)$.

24. Genèse 21,14 est, selon Reis, la preuve, non pas d'une séparation géographique entre Hagar et Abraham, mais d'une rupture maritale. Le verbe "renvoyer » dans le verset renvoie, selon elle, au divorce. 


\section{8 - ARCHIVES DE SCIENCES SOCIALES DES RELIGIONS}

Hagar occupe bel et bien le rôle d'une épouse à part entière ; ce n'est donc pas à la domestique étrangère qu'Abraham s'unit, mais à cette femme nommée Hagar : " on voit, écrit Wénin, qu'Abram tout en jouant le jeu initié par Sarai, n'adopte pas tout à fait sa façon de traiter la domestique en objet. Il semble plutôt considérer celle-ci comme une personne vers qui il vient avec un certain respect. " $(2001: 43)^{25}$. Hagar s'affirme ainsi, pour la première fois, comme une épouse légitime dont le statut est supérieur à celui de celle qui l'a prise comme moyen de se satisfaire. Ce renversement de situation, souvent lu comme un geste arrogant et méprisant d'Hagar envers Sara, se prête néanmoins à une autre lecture. Car, selon Wénin, la fin de Genèse 16,4 (« et elle [Sarai] fut légère à ses yeux ») n'est pas aussi claire qu'on le croit. Une lecture alternative permet d'élucider le côté subjectif de l'histoire où le possessif de « ses yeux » pourrait renvoyer non à Hagar, mais à Sara même.

Dans cette lecture alternative de la Genèse, non seulement Hagar occupe la position positive de femme d'Abraham, mais elle représente également un personnage biblique central ${ }^{26}$. Ainsi, Nikaido (2001) montre, dans une perspective intertextuelle, comment Hagar symbolise la figure féminine de l'alliance avec le divin ${ }^{27}$. Dans le même ordre d'idées, Jarrell (2002) estime que la relation entre Hagar et Dieu informe et sert de prototype à l'ensemble des relations contractuelles entre les femmes de la Genèse et Yahweh. Cette relation contractuelle est exprimée dans le récit de la naissance ; ce genre narratif représente la contrepartie de l'alliance divine avec les hommes ${ }^{28}$. L'histoire d'Hagar, comme le note Dozeman (1998), sert aussi de modèle pour la vie de Moïse et préfigure la souffrance et l'esclavage d'Israël ${ }^{29}$. Et l'ensemble des deux histoires montre comment Dieu

25. Comme le note Leviant (1999), bien que le terme hébreux, isha, pour désigner Hagar dans Genèse 16,3, signifie épouse et non concubine, c'est plutôt ce dernier terme qui en est la traduction la plus courante.

26. Comme le montre Nikaido, Hagar ainsi que son fils Ismaël sont associés à des figures bibliques centrales. Celle-là est associée à Hannah, celui-ci à Samuel ainsi qu'à Joseph, petitfils d'Isaac et fils de Jacob (2001: 237).

27. Selon Nikaido, c'est Hagar, et non Sara, qui représente l'homologue féminin du patriarche Abraham et son véritable compagnon dans les tribulations. "Therefore, in contrast to the negative position she occupies opposite Sarah, Hagar is surrounded with attributes that make her Abraham's true "companion" in tribulation and fitting counterpart with regard to his patriarchal role » (Nikaido, $2001: 228$ ).

28. L'intimité entre l'Éternel et Hagar était perçue comme insupportable, et c'est pourquoi on procéda à ce que Jarrell appelle le «nettoyage patriarcal ». L'auteur n'exclut pas la possibilité d'une restructuration des récits primitifs par les rédacteurs patriarcaux, par l'insertion d'un intermédiaire contractuel, un mari notamment. Selon Jarrell, ce genre de réarrangement est bien perceptible dans l'histoire d'Hagar; surtout si l'on compare la version J, (Genèse, 16,10), où la promesse lui est directement adressée (L'ange de l'Éternel lui dit : Je multiplierai ta postérité, et elle sera si nombreuse qu'on ne pourra la compter), à la version E, (Genèse, 21,13), où la promesse est plutôt adressée à Abraham (Je ferai aussi une nation du fils de ta servante; car il est ta postérité).

29. En effet, les similitudes entre l'histoire de Hagar, dans Genèse 16 et 21, et de Moïse, dans Exode 1-15, sont très révélatrices. 
transforme un conflit familial en une occasion de libération fondatrice des nations ${ }^{30}$. De toutes ces recherches récentes, on pourrait avancer que le rôle négatif souvent assigné à Hagar dans certaines sources post-bibliques, s'est constitué à l'encontre de l'esprit du texte sacré. Il s'agit d'une inversion symbolique qui concerne la position d'Hagar en tant que deuxième femme d'Abraham et mère de son premier fils.

Considérons à présent la tradition arabo-islamique. Tandis que la référence coranique à Sara est plus ou moins explicite ${ }^{31}$, le nom d'Hagar et les épisodes de sa vie évoqués par le récit biblique sont pareillement tus. Cette occultation se perpétue, pendant une longue période, dans la tradition musulmane. Ce n'est que progressivement qu'Hagar sera connue par son nom propre. Pour Benslama (2002), les raisons de cette absence ont vraisemblablement un rapport avec l'origine servile de la mère d'Ismaël, qui de plus fut répudiée et chassée par celui dont on veut faire le Père de tous les musulmans. D'autant plus que juifs et chrétiens de la région ne se privaient pas de rappeler aux Arabes leur ascendance "impure".

En effet, la servitude d'Hagar était au cœur des débats entre musulmans et non-musulmans. Ainsi, l'historiographe Mas'ûdi ( $\mathrm{X}^{\mathrm{e}}$ siècle) mentionne que l'empereur byzantin Nicéphore le Logothète (802-811) de la dynastie isaurienne a dû prendre un décret où il défend à ses sujets d'appeler les Arabes des Sarrasins, nom qui signifiait " esclaves de Sara », et que les Grecs leur donnaient par allusion injurieuse à Hagar (Dagorn, 1981: 202). La servitude de celle-ci n'était pas uniquement un point de controverse entre musulmans et non-musulmans, il semble qu'elle était aussi un enjeu de débat entre musulmans arabes et musulmans non arabes (Dagorn, 1981 ; Benslama, 2002 ; Kaltner, 2002). Face à l'origine agarienne et ismaélienne des premiers, les Persans et les Nabatéens vont se prévaloir d'une ascendance abrahamique par Isaac, traitant les Arabes de fils de la lakhnâ, la puante ${ }^{32}$.

Les Arabes, nonobstant les origines serviles d'Hagar, vont finir par l'adopter comme "ancêtre-mère ", la défendant outrageusement contre ses détracteurs.

30. "Genesis 16 and 21, écrit Dozeman, trace the transformation of Hagar from slave to founder of the Ishmaelites in a continuous story, which provides the prototype for Moses, who undergoes similar transformation in the wilderness to become founder of the Israelite nation [...]. It is her son, Ishmael, who mirrors Israel's wilderness experience » (1998: 33).

31. Sara est désignée dans le Coran par "femme d'Abraham ». On lit, par exemple, dans la sourate 11,71-72: "son épouse [d'Abraham] était restée présente. Or elle éclata de rire, car Nous lui avions transmis la bonne nouvelle d'Isaac et, après Isaac, de Jacob. Inoui !, dit-elle, j'enfanterais, alors que je suis une vieille, et mon époux, que vous voyez, en tant que cheikh ? Ce serait vraiment chose étonnante ".

32. La question de la shu'ûbiya est très connue dans l'histoire musulmane, surtout à l'époque des Abbasside. Une grande production littéraire, la poésie notamment, en atteste. Pour plus de détails sur cette querelle entre les Arabes et les $s h u^{\prime} \hat{u} b \hat{\imath}$ (musulmans non arabes), voir Dagorn, op. cit. : 220-224. 
René Dagorn rapporte un exemple qui illustre bien cette nouvelle posture : il s'agit de la réplique d'Abû Yahyâ Ibn Mas'ada aux dénigreurs de la mère d'Ismaël : «Il [Abraham] a choisi Hagar de préférence à votre mère (Sara) comme dépositaire de son message et la prit pour concubine, alors qu'il était déjà enfant, celle qui reçut (en dépôt) notre père Ismaël, que Dieu répande sur lui ses bénédictions " (1981: 229). Reprenant le récit de la Genèse concernant Hagar, Abû Yahyâ l'enrichit d'éléments nouveaux visant à magnifier le rôle de la mère d'Ismaël et à déprécier les avantages de Sara. Ce que l'on va désormais mettre en avant, c'est son statut d'épouse d'Abraham, noble et pure de surcroît. Ibn Qutayba, entre autres, s'attache à le montrer avec les expressions les plus claires. Une femme comme Hagar, écrit-il, est purifiée par Dieu de toute souillure, parfumée de toute mauvaise odeur, épouse agréé de l'ami de Dieu Abraham et mère de deux hommes vertueux qui sont Ismaël et Mohammed (Dagorn, 1981 : 223).

Parallèlement à cette reconsidération positive de la mère d'Ismaël, on assiste à une dépréciation de Sara. Celle-ci est accusée de cruauté à l'endroit d'Hagar et tenue pour seule responsable du déchirement de la grande famille d'Abraham. Al-Tabari exprime cette tendance dans un passage incisif : « Lorsque Agar accoucha d'Ismaël Abraham fut rempli de joie; mais Sara éprouva de la colère et une violente jalousie. N'étant plus maîtresse d'elle-même, elle eut des querelles et des disputes avec Abraham, et elle lui dit des injures. Ensuite elle dit avec serment : Je couperai une partie quelconque du corps d'Agar, ou une main, ou un pied, ou une oreille, ou le nez. Mais après avoir réfléchi, elle dit : C'est moi qui ai commis cette faute, car j'ai donné Agar à Abraham. Il ne serait pas juste de couper à cette fille une partie de son corps, ni de la tuer ; mais j'ai juré, et il faut absolument que je lui coupe quelque chose. Après y avoir pensé, elle dit : Je la circoncirai pour l'empêcher de rechercher les hommes " (2003: 80). Selon alTabari, lorsque Sara eut excisé Hagar, Dieu imposa la circoncision à toute la famille d'Abraham, hommes et femmes, de sorte que Sara elle-même fut obligée de se circoncire alors qu'elle avait 70 ans.

Tout se passe comme si l'excision d'Hagar, voulue comme une humiliation et une mutilation, se situait à l'origine de l'alliance par circoncision. D'après l'exégète Ibn Kathîr (XIV siècle), Hagar fut la première femme aux oreilles percées et la première femme circoncise. L'auteur use de la métaphore "thaoulat daylaha », qui signifie littéralement : "sa queue s'allongea ». Fekkar donne une interprétation intéressante à cette expression sibylline : "elle [Hagar] y gagna en puissance, ce que, dans la perspective religieuse, nous entendons par "celui qui est rabaissé sera élevé » (1988: 238-239).

Les signes de cette élection sont aussi manifestes dans l'expérience du désert. Bien que le Coran ne donne aucune information au sujet de l'expulsion d'Hagar, les commentateurs tardifs, influencés par les sources juives, adopteront la version biblique, mais avec un déplacement assez révélateur. Abraham n'envoie pas Hagar et Ismaël seuls dans le désert, mais il les accompagne à l'enceinte sacrée 
de Dieu, la Mecque. Un autre détail important : le père maintiendra le lien avec sa femme et son fils, durant toute sa vie, en leur rendant visite régulièrement (alTabari, 2003 ; Ibn Qutayba, 1969 ; Dagorn, 1981 ; Kaltner, 2002). Dans cette expérience du désert, Dieu intervient non seulement pour sauver Hagar et son fils Ismaël d'une mort certaine, mais il fait d'elle la première personne qui initiera un rituel musulman lié au pèlerinage, celui de la course entre les collines de Safâ et Marwa.

\section{Héritage et descendance}

Le revirement dans le comportement de Sara envers Hagar et son fils Ismaël montre clairement le facteur principal mis en jeu dans le réseau des relations liant l'ensemble des membres de la famille du Patriarche. Genèse 21,9-10 laisse entrevoir le rôle important que joue l'héritage dans le conflit qui oppose Sara à Hagar. Plusieurs auteurs (Hackett, 1989 ; William, 1993) confirment le rôle décisif de l'héritage dans l'expulsion d'Ismaël et sa mère. Sur ces motivations économiques de Sara, Ann Hackett écrit : " the original story [en référence à Genèse 21,9] must have said that he [Ismaël] did something to rile Sarah, to make her think or to remind her that he was also in a position to inherit " $(1989: 20)$.

Delores S. William écrit, toujours en référence à Genèse 21,9-10 : « Economic realities, specifically inheritance, are the central issues here. Hagar is poor, and apparently Sarah does not want Hagar's station elevated, as it no doubt would be if Ishmael received the inheritance from his father that the firstborn son was supposed to receive. According to early Hebrew custom, and Assyrian and Nuzi law, the eldest received a double portion of his father's wealth. Law forbade the father from showing special privilege to the son of the wife he preferred and thereby protected the firstborn son's inheritance rights » (1993: 27).

Certaines lectures juives vont dans ce sens ; Ismaël, selon le Midrash, aurait indécemment réclamé le droit, en tant qu'aîné, de recevoir une part double de l'héritage (Reis 2000 : 94) ${ }^{33}$. Bien que le récit biblique ne donne aucun indice de la portée péjorative du geste d'Ismaël, certaines interprétations juives classiques l'ont exagérée au point de l'assimiler à un acte immoral grave : l'idolâtrie et le meurtre (Zucker, 1990 : 40 ; Reis, 2000 : 94), acte qui pourrait irrémédiablement priver Ismaël de tout droit à l'héritage ${ }^{34}$.

33. "We do not have to wonder, écrit Reis, why Ishmael is to be disinherited. He has became tipsy at the feast [...]. He is of the right age to be curious, to experiment and to make a spectacle of himself. Foolishly, rather than hiding his impairment, he might have shown it off in a puerile attempt to look sophisticated. Sara may even have pointed out to Abraham that Ishmael takes after his cousin, Lot. Abraham distanced himself from Lot, and Sarah demands the removal of this other bad influence " (2000:97).

34. Le droit d'aînesse d'Ismaël est gênant au point même que sa filiation avec Abraham soit controversée. Römer (1999 : 172) cite l'avis du Rabbi 'Awira qui présume qu'Abraham, lors du festin eschatologique, aurait dit : « je ne peux pas rendre grâce car j'ai engendré Ismaël ». 
Comme on l'a mentionné pour Hagar, ou pourrait affirmer que les interprétations négatives de la figure ismaélienne vont à l'encontre de l'esprit du texte biblique (Zucker, 1990 ; Leviant, 1999 ; Römer, 1999 ; Kaltner, 2002) ${ }^{35}$. On pourrait parler, dans ce cas, d'une inversion symbolique qui concerne le droit d'aînesse d'Ismaël et ses conséquences en matière d'héritage. L'héritage dont il est question ici est plus un capital symbolique et spirituel. C'est l'héritage de toute une tradition prophétique qui représente une alliance avec le divin. Aussi, le juif cherchant à défendre le droit d'Isaac à l'héritage abrahamique, contre celui d'Ismaël, tente-t-il de légitimer son propre héritage de cette alliance avec le divin. Le musulman essaie tout autant de conforter la position sociale de la mère d'Ismaël pour pouvoir prétendre au même titre que le juif à cet héritage (fig. 3).

$$
\begin{gathered}
\Sigma \text { Agar = servante }(-) / \text { Ismaël = aîné }(+) \rightarrow \sum \text { Sara } \\
\text { = épouse légitime }(+) / \text { Isaac }=\operatorname{cadet}(-)
\end{gathered}
$$

Fig. 3a : la version juive. Dans ce cas, l'ensemble $(\Sigma)$ des charges positives $(+)$ et négatives (-) s'annule. Ainsi pour que la valeur finale soit positive en faveur d'Isaac, d'autres paramètres sont intégrés à cette équation, l'immoralité d'Ismaël par exemple. Isaac deviendrait ainsi le seul héritier de la promesse.

$$
\begin{aligned}
\Sigma \text { Sara } & =\text { première épouse }(+) / \text { Isaac }=\text { cadet }(-) \rightarrow \Sigma \text { Agar } \\
& =\text { deuxième épouse }( \pm) / \text { Ismaël }=\text { aîné }(+)
\end{aligned}
$$

Fig. 3b : la version musulmane. Dans ce cas, il est inutile d'ajouter d'autres facteurs pour que la valeur positive finale soit en faveur d'Ismaël; il suffit de neutraliser $( \pm)$ la charge négative attribuée à Agar dans l'équation précédente par un simple déplacement de son rôle de servante à celui d'épouse.

\section{Conclusion}

Dans les deux religions, judaïque et islamique, Abraham est considéré comme un père. Toutefois une conception différente de cette appartenance les sépare. Dans le judaïsme, la généalogie abrahamique reste absolument nécessaire à la représentation religieuse où l'Isaac et l'Israël spirituels ont remplacé l'Isaac et

35. Selon Zucker, ce sont les interprétations tardives, surtout dans la littérature talmudique et certaines exégèses du Moyen Âge, qui ont assigné un rôle très négatif à Ismaël, sa mère et leurs descendants. "The normal tensions, écrit-il, with their Arab or Muslim neighbours, whether in Jerusalem or Hebron, the Galilee or elsewhere, were often expressed with reference to Biblical events. For example, the medieval classical Jewish commentators Rashi, Ibn Ezra and Ramban, all state that Ishmael's descendants will be troublesome and aggressive. Ramban notes that, after initial successes, in the end they will be defeated. " (1990:38-39). 
l'Israël charnels. Ainsi, le judaïsme se veut l'héritier non seulement spirituel, mais également physique du Patriarche. Dans le Coran, la descendance par Isaac n'est pas moins marquée que celle par Ismaël; le don prophétique de celui-ci ne dépasse aucunement le legs de celui-là. Cette remarque est soutenue par plusieurs versets où Isaac est toujours associé à son frère Ismaël. Même la descendance d'Isaac, Jacob entre autres, est directement liée à Ismaël. Il est dit, par exemple, dans la sourate 2,133 : " auriez-vous été témoins du moment où la mort pressa Jacob ? Il a dit à ses fils: "Qu'adorerez-vous après moi ?" Ils dirent: "Nous adorerons ton Dieu et le Dieu de tes pères, Abraham, Ismaël et Isaac. C'est un Dieu unique. À lui nous nous soumettons».

Dans d'autres versets, le Coran insiste sur le caractère non hiérarchique entre les prophètes et en fait un acte de foi. Ainsi dans la même sourate 2,136, il est écrit : "Dites : "Nous croyons en Dieu et en ce qui est descendu sur nous, en ce qui est descendu sur Abraham, Ismaël, Isaac, Jacob, les lignages, en ce qui fut donné à Moïse, à Jésus, fut donné aux prophètes de la part de leur Seigneur. De tous ceux-là nous ne séparons pas un seul, puisque c'est à Lui que nous nous soumettons".

Il est évident qu'Ismaël, dans le Coran, ne constitue pas une revendication pro-arabe contre les juifs. Les musulmans sont intégrés dans la communauté des croyants dont le père est un Abraham et Dieu est unique. Aussi la symbolique et le sens du déplacement d'Isaac vers Ismaël, et qui concerne aussi Hagar, sontils à rechercher, non pas dans le Coran, mais dans la tradition musulmane postcoranique. Ceci indique que certaines transformations qui concernent Ismaël se sont faites en opposition avec l'esprit du texte sacré. La signification de ces déplacements, comme le fait remarquer Bonte (1999), déborde l'effet de légitimation généalogique du prophète de l'islam et de l'ensemble des Arabes. Les dimensions politiques et idéologiques de toutes ces transformations, dans un sens comme dans l'autre, sont indéniables. La tension, inventée, entre Ismaël et Isaac devient alors un paradigme de la tension entre juifs et Arabes, qui est encore vive aujourd'hui dans le conflit douloureux qui oppose Israéliens et Palestiniens (Zucker, 1990 ; Römer, 1999).

Judaïsme et islam ont chacun élaboré leur version de l'arbre généalogique d'Abraham, chargeant de connotations positives ou négatives Sara et Hagar, Isaac et Ismaël. Hagar la servante étrangère devient l'épouse noble et pure. Ismaël est l'aîné, mais Isaac est l'élu. Celui-ci a tendu le cou au couteau ; celui-là l'a tendu aussi et, de plus, a agonisé dans le désert. L'un comme l'autre ont été sauvés in extremis par la volonté de Dieu et à travers eux toutes leurs descendances respectives. Tout se passe comme si « des contemporains s'affrontent par passés interposés en se choisissant leurs ancêtres. Le sens de la tradition s'inverse alors, elle devient une rétrospection camouflée : ce sont les fils qui engendrent 
44 - ARChives DE SCIENCES SOCIALES DES RELIGIONS

leurs pères pour justifier les changements réels qu'ils apportent au système existant» (Pouillon, 1993 : 47).

Zakaria RHANI ${ }^{36}$

Université de Montréal - Département d'anthropologie

zrhani@gmail.com

\section{Bibliographie}

ABDESALAm Sadok, 1999, "Le sang du sacrifice chez les Soudanais de Wad Madani » in Bonte P., Brisebarre A.-M., Gokalp A., Sacrifices en Islam: espace et temps d'un rituel, Paris, CNRS Éditions, pp. 355-381.

Al-TABARI Mohammed Ibn Jarir, 2003, Histoire des Prophètes et les rois : de la création à la dernière Révélation (trad. Hermann Zotenberg), Paris, Éditions de la Ruche.

-, 2005, Tarîkh al-Tabari (I) (Chronique d'al-Tabari), AL-QAYSSI Iyyad (éd., comment.), Beyrouth, Dar Ibn Hazm.

Benslama Fethi, 2002, La psychanalyse à l'épreuve de l'islam, Paris, Aubier. Bible (La), 1956, (trad. École Biblique de Jérusalem), Paris, Éditions du Cerf.

Bible (La), 1979, (trad. Louis Segond), Genève, Nouvelle Édition de Genève.

BONTE Pierre, 1999, « Sacrifice en Islam. Textes et contextes » in Bonte P., Brisebarre A.-M., Gokalp A., Sacrifices en Islam: espace et temps d'un rituel, Paris, CNRS Éditions, pp. 21-61.

ClÉment Catherine, 2002, Claude Lévi-Strauss, Paris, PUF, Coll. «Que sais-je ».

Coran (Le), 1995 [1900], (trad. Jacques Berque), Paris, Albin Michel.

DAGORN René, 1981, La geste d'Ismaël d'après l'onomastique et la tradition arabes, Genève, Droz.

Dozeman Thomas B., 1989, "The wilderness and salvation history in the Hagar Story ", Journal of Biblical Literature, 117-1, pp. 23-43.

FeKKar Yamina, 1988, " Le mythe de Hajar ", Peuples méditerranéens, 44-45, pp. 235-245.

Goody Jack, 1979, La raison graphique, Paris, Éditions de Minuit.

HACKeTT Ann J., 1989, « Rehabilitating Hagar: Fragments of an Epic Pattern » in Day P.L., Gender and Difference in Ancient Israel, Minneapolis, Fortress Press, pp. 12-27.

IBN QuTAYBA Abou Mohammed Ibn Muslim, 1969, al-Ma'ârif (Les Connaissances) 'UKÂCHA Tarouat, (éd. présent.), Le Caire, Dâr al-Ma'ârif.

JARRELL R.H., 2002, "The birth narrative as female counterpart to covenant ", Journal for the Study of the Old Testament, 97, pp. 3-18.

KALTNER John, 2002, "Abraham's Sons: how the Bible and the Qur'an see the same story differently », Bible Review, 45-46, 18-2, pp. 16-23.

LEVIANT Curt, 1999, "Parallel lives: the trials and traumas of Isaac and Ishmael », Bible Review, 47, 15-2, pp. 20-25.

LÉvi-STRAuss Claude, 1974 [1958], Anthropologie structurale, Paris, Plon.

-, 1996 [1973], Anthropologie structurale deux, Paris, Plon.

-, 1983, Le Regard éloigné, Paris, Plon.

36. L'auteur tient à remercier Jean-Claude Muller, Gilles Bibeau et Robert Crépeau pour leurs encouragements et leurs lectures critiques et pertinentes. 
LES RÉCITS ABRAHAMIQUES DANS LES TRADITIONS JUDAÏQUE ET ISLAMIQUE - 45

Menasce Jean (de), 1951, "Traditions juives sur Abraham », Cahiers Sioniens, Abraham: père des croyants, 2, pp. 96-103.

Millar Fergus, 1993, "Hagar, Ishmael, Josephus and the Origins of Islam », Journal of Jewish Studies, 44-1, pp. 23-45.

NIKAIDO Scott, 2001, "Hagar and Ishmael as literary figures: An intertextual study », Vetus Testamentum, 51-2, pp. 219-242.

PABST Irene, 2003, « The interpretation of the Sarah-Hagar-stories in rabbinic and patristic literature. Sarah and Hagar as female representations of identity and difference ", Lectio difficilior, 1.

Pouillon Jean, 1993, Le cru et le su, Paris, Éditions du Seuil.

REIS, Pamela Tamarkin, 2000, " Hagar requited », Journal for the Study of the Old Testament, 87, pp. $75-109$.

RÖMER Thomas Christian, 1999, "Isaac et Ismaël, concurrents ou cohéritiers de la promesse ? Une lecture de Genèse 16 ", Études Théologiques et Religieuses, 74-2, pp. 161-172.

WéNIN André, 2001, "Sarai, Hagar et Abram. Une approche narrative et contextuelle de Gn 16,1-6 », Revue Théologique de Louvain, 32, pp. 24-54.

Williams Delores S., 1993, Sisters in the Wilderness, Maryknoll, Orbis Books.

WYATT Nick, 1996, "Les mythes des Dioscures et l'idéologie royale dans les littératures d'Ougarit et d'Israël ", Revue Biblique, 103-4, pp. 481-516.

ZUCKER David J., 1990, "Conflicting conclusions: the hatred of Isaac and Ishmael ", Judaism, 39-1, pp. 37-46.

\section{Résumé}

Ce texte représente une analyse structurale du mythe d'Abraham. En suivant les transformations que cette histoire a subies en passant d'une tradition religieuse à une autre (essentiellement juive et musulmane), on démontre comment l'ensemble des relations qui composent le récit du Patriarche (circoncision/sacrifice, épouse/servante, aînesse/héritage) suit une évolution symétrique mais opposée. Judaïsme et islam ont chacun élaboré leur version de l'arbre généalogique d'Abraham, chargeant de connotations positives ou négatives les composantes de ce mythe (Sara et Hagar, Isaac et Ismaël).

Mots-clés : mythe, structure, Abraham, judaïsme, islam.

\section{Abstract}

This essay presents a structural analysis of the myth of Abraham. Having examined the transformations that it underwent as it passed from one religious tradition to another (primarily Jewish and Muslim), I argue that the relations around which the myth of the Patriarch is built (circumcision/sacrifice, wife/handmaid, firstborn/ inheritance) follow a symmetrical albeit opposite evolution. Judaism and Islam developed their own versions of Abraham's family tree, each one charging its genealogical episodes with positive or negative connotations (ex. Sarah and Hagar, Isaac and Ishmael).

Key words: myth, structure, Abraham, Judaism, Islam. 
46 - ARChives De SCIENCES SOCIALES DES RELIGIONS

\section{Resumen}

Este rexto representa un análisis estructural del mito de Abraham. Siguiendo las transformaciones que esta historia ha sufrido en el pasaje de una tradición religiosa a otra (esencialmente judía y musulmana), se muestra cómo el conjunto de las relaciones que componen el relato del Patriarca (circuncisión/sacrificio, esposa/sirviente, mayorazgo/herencia) sigue una evolución simétrica pero opuesta. Judaísmo e islam han elaborado cada uno su propia visión del árbol genealógico de Abraham, cargando de connotaciones positivas o negativas a las componentes de este mito (Sara y Hagar, Isaac e Ismael).

Palabras clave: Mito, estructura, Abraham, judaísmo, islam. 